\title{
ROLES AND SCOPE OF SYSTEM INTERFACE IN INTEGRATED CONTROL SYSTEM FOR MULTI DISASTER COUNTERMEASURE
}

\author{
SEUNGHWA PARK \& CHANGHEE HONG \\ Multi Disaster Countermeasure Organization, Korea Institute of Civil Engineering and Building Technology.
}

\begin{abstract}
Damages on human and physical properties have recently become significant due to natural disasters, such as earthquake and hurricanes, as well as social disasters, such as fire in subway and terrorist attacks using explosive. Notably, as buildings are becoming taller and more complex, demands on countermeasure to multi disasters, such as earthquake, fire, and flooding, are increasing for complex skyscraper facilities. Complex skyscraper facilities are equipped with constant management systems, such as facility management, lighting management, CCTV management, and parking management, as well as event-based emergency management system, such as fire control, flooding control, seismic detection, and wind detection. While part of the information for each system is currently being collected by a central monitoring system in the form of SI (system integration), it is difficult to perform SOP (standard operating procedure)-based integrated control because data protocols for sensors and receivers are different. An integrated system for efficient countermeasure/evacuation in complex skyscraper facilities is currently under development, and this study organizes the roles and scope of system interface for linkage of information among the integrated system, disaster information collection system, analysis system, and countermeasure system. The scope of disaster at this point was limited to earthquake, flooding, and fire. The system interface that is designed by this study is one of core modules for implementing an integrated system, and is to be used to present future directions for development of integrated CPS (Cyber Physical System) in countermeasure to disasters.

Keywords: BIM-GIS platform, dashboard, multi disaster, SOP, system interface
\end{abstract}

\section{INTRODUCTION}

A convergence research group on disaster response has been launched to develop and practically use an integrated system to protect massive complex facilities such as skyscrapers against earthquakes, fire, flood and other disasters within 3 years. In Korea, there are currently meters such as earthquake accelerometers in operation at around 600 major national facilities, but effective disaster response has been difficult due to the absence of a system and operating body for integrated management of the related information and data. Amid this situation, the Disaster Response Research Group conducted a project to develop an integrated information platform, with the aim of predicting the risk factors associated with disasters and calamities and ensuring a fast response and recovery, in the event of an accident, by promptly collecting and analysing the necessary information. The plan is to develop the key technology for each element of the platform in the first and second years, and to set up a testbed in the third year to perform a wide variety of verification tests. At present, the project is in its first year. Approximately 24 million dollars will be invested into this 3-year convergence research project, which will be undertaken by some 200 researchers at the Korea Institute of Civil Engineering and Building Technology (KICT). The research procedure is as follows (Fig. 1):

The disaster information collection system stage involves implementation of integrated measuring and monitoring technology and establishment of an information collection system to create an information system for responding to disasters such as fire, earthquake, and flood. 


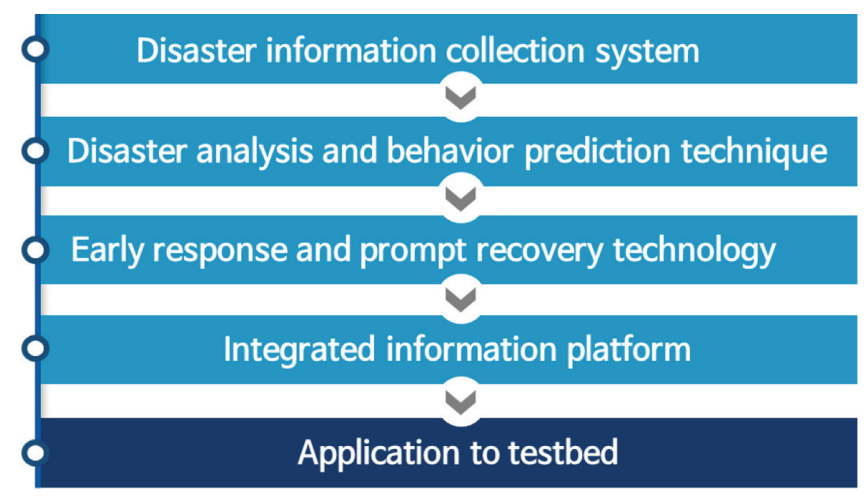

Figure 1: Research procedure.

In the disaster analysis and prediction stage, simulations of a combination of disasters will be performed to predict the risk factors and formulate response and recovery measures, after which the prediction techniques will be set forth.

In the response and recovery stage, an early response and evacuation support system and a prompt response and recovery manual will be established so as to minimize damage and secure the golden hour.

For the integration and control of the aforementioned systems, an integrated information platform will be developed by setting up an integrated CPS based on an open platform server so as to ensure high reliability in information delivery and management.

Lastly, the integrated CPS will be applied to the testbed, and an ROI (return on investment) analysis will be carried out to assess the extent to which the practical use of the system is possible.

This study was conducted on the system interfaces for the BIM/GIS platform ${ }^{1}$ [1] server, analysis system, and sensors and measuring systems in the integrated information platform development stage for the purpose of assessing the role and scope of each of the system interfaces comprised of modules.

\section{INTEGRATED INFORMATION PLATFORM FOR DISASTER RESPONSE}

The integrated information platform for disaster response consists of a BIM/GIS platform server and system interfaces. In this particular chapter, the significance of each system will be examined.

\subsection{BIM/GIS Platform Server}

One of the limitations of 3D spatial information services such as Google Earth and V-World is that they only present the external appearances of buildings and structures. Since we spend $70 \%$ of our time indoors, however, there is a need for technology that can capture and present both the outside and inside of buildings and structures. With respect to this matter, the National Institute of Building Sciences (NIBS) and National BIM Standard (NBIMS) have stated that indoor and outdoor spatial information can be integrated together, centring on the contact points of BIM and GIS [2].

1 It means the Platform that BIM(Building Information Modelling) data and GIS(Geographic Information System) data can be operated together on one system. It is simply called "BIM on GIS Platform" or "BIM/GIS Platform". 


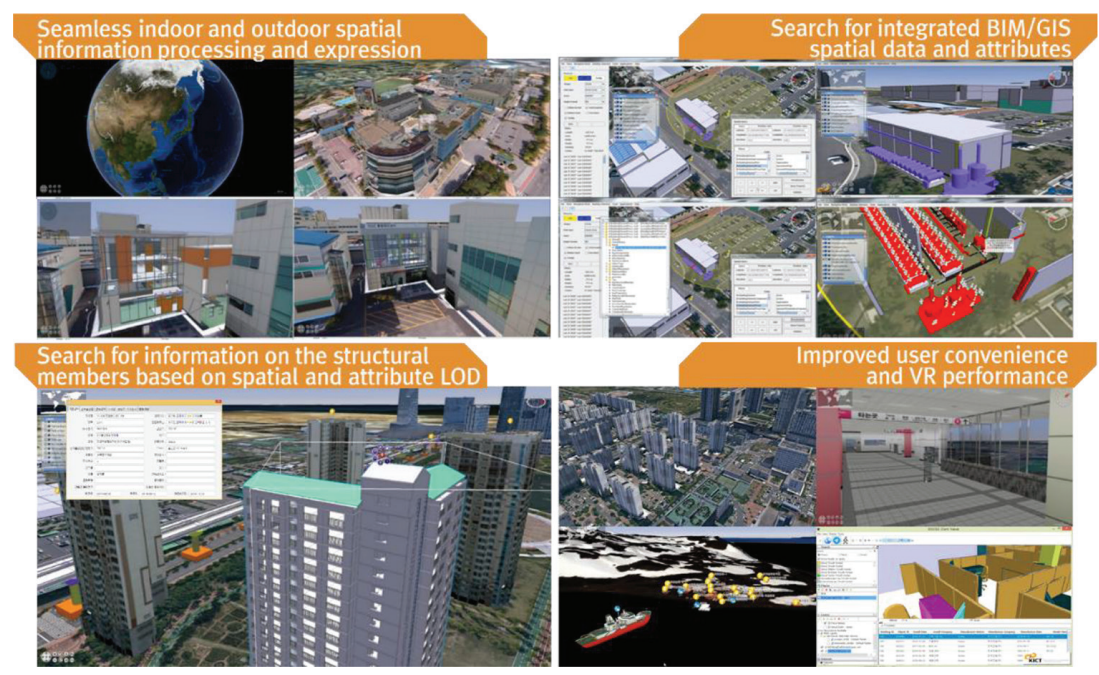

Figure 2: Main functions of BIM/GIS platform [3].

Accordingly, a platform characterized by BIM/GIS interoperability was developed to create a database of attributes on a BIM model and to enable a seamless service on indoor and outdoor spatial information. Such platform, which was developed by our team, allows seamless use and management of massive amounts of 3D BIM and GIS data on a large number of buildings within a city or a regional sphere simultaneously, rather than a single building.

The BIM/GIS platform can express both the indoor and outdoor spatial information. In the case of attributes related to disaster management and prevention, for instance, it can be used to express the information on the bearing and non-bearing structures, building materials, and evacuation. In addition, it can be used for disaster information analysis, facility management, as well as fire-fighting training such as XVR [4]. For this reason, it serves as the core platform of the integrated system.

\subsection{System Interfaces}

As shown in Figure 3, the system interfaces collect and accumulate information in connection with the BIM/GIS platform server, described in Section 2.1, as well as various measuring systems, video streamer, access controller, and analysis system. They are synchronized with a support system, and are responsible for primary response and alarm control.

Each interface module plays a set of unique roles depending on the field of response and its composition. The role and scope of the system interface will be described in Chapter 3 .

\section{THE ROLE AND SCOPE OF THE SYSTEM INTERFACES}

\subsection{The role of the system interfaces}

The system interfaces performs diverse roles, in connection with the platform, as shown in Figure 4. 


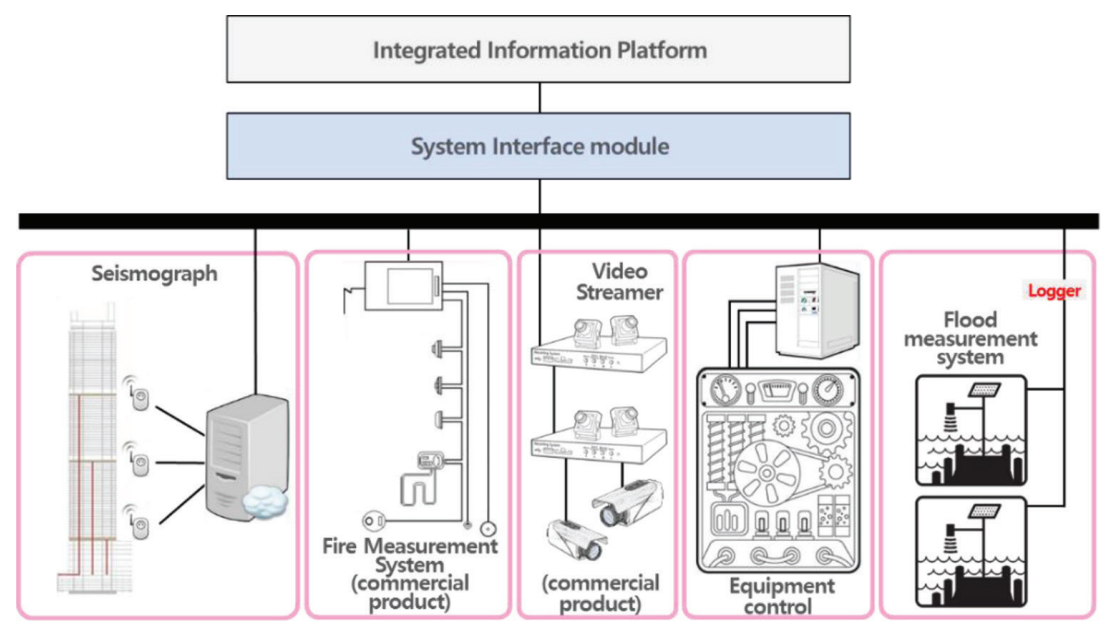

Figure 3: Concept of system interface's architecture.

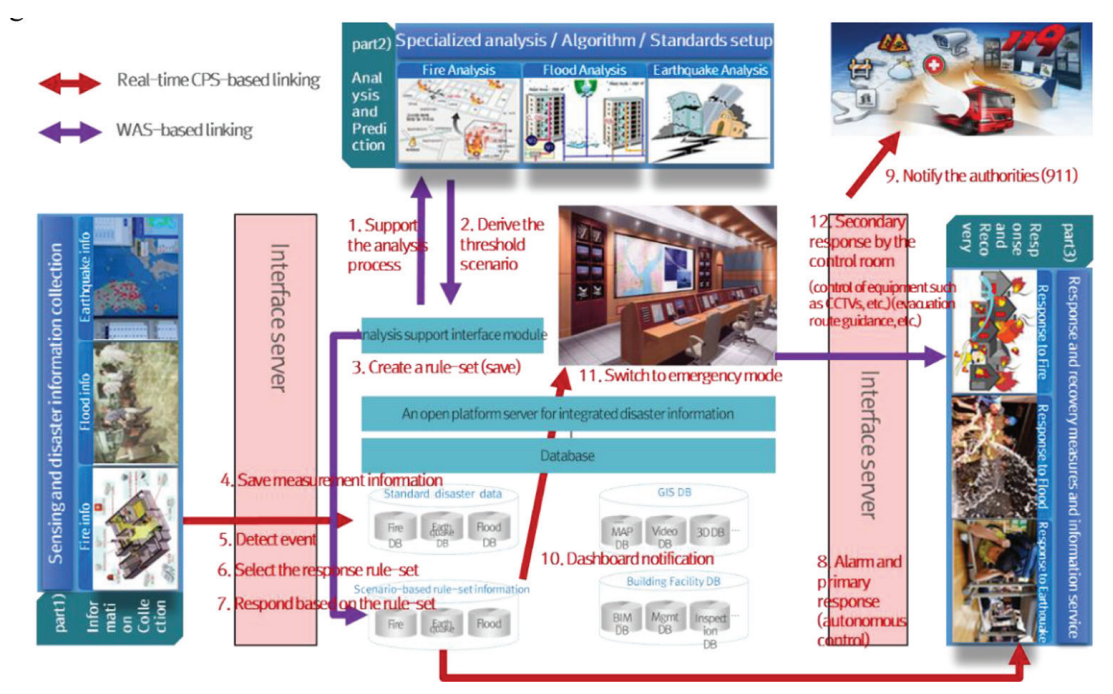

Figure 4: Roles of interface modules.

The disaster information collection interface, for example, stores measuring information, detects events, and selects a response rule-set for optimum response, based on which the instructions for a response are given. In the case of the analysis and prediction interface, it supports the collected disaster information to be communicated to the analysis system, and saves the threshold scenarios derived from the analysis system in the scenario-based rule-set database. As for the response and recovery interface, it supports autonomous control for the primary response and the secondary responses such as control of equipment including CCTVs and evacuation route guidance.

The cases requiring real-time high-reliability data are indicated with a red arrow, whereas data requiring WAS (Web Application Server)-based stability and security are indicated with a purple arrow. 
Table 1: Scope of each system interface module.

\begin{tabular}{|c|c|c|c|}
\hline $\begin{array}{l}\text { Module } \\
\text { name }\end{array}$ & $\begin{array}{l}\text { Analysis sys- } \\
\text { tem-linked } \\
\text { information module }\end{array}$ & $\begin{array}{l}\text { Measurement infor- } \\
\text { mation-linked } \\
\text { interface module }\end{array}$ & $\begin{array}{l}\text { Autonomous con- } \\
\text { trol-linked interface } \\
\text { module }\end{array}$ \\
\hline $\begin{array}{l}\text { Descrip- } \\
\text { tion }\end{array}$ & $\begin{array}{l}\text { - Identifies spatial } \\
\text { information to } \\
\text { support the analyses } \\
\text { of disasters (fire, } \\
\text { earthquake, or } \\
\text { flood) occurring in } \\
\text { super-tall complex } \\
\text { facilities } \\
\text { - Processes spatial } \\
\text { information to } \\
\text { support the disaster } \\
\text { analysis system } \\
\text { - Link with the } \\
\text { integrated informa- } \\
\text { tion platform and } \\
\text { the analysis system } \\
\text { for disaster (fire, } \\
\text { earthquake, or } \\
\text { flood) response }\end{array}$ & $\begin{array}{l}\text { - Receive measure- } \\
\text { ment information in } \\
\text { real time } \\
\text { - Identify the thresh- } \\
\text { olds based on the } \\
\text { real-time measure- } \\
\text { ment information } \\
\text { (fire, earthquake, or } \\
\text { flood) } \\
\text { - Select the risk } \\
\text { scenarios connected } \\
\text { with event signals } \\
\text { - Connect to the } \\
\text { authorities (emer- } \\
\text { gency call, 911) }\end{array}$ & $\begin{array}{l}\text { - Call the rule-set } \\
\text { database for equipment } \\
\text { control } \\
\text { - Perform automatic } \\
\text { equipment control and } \\
\text { response based on the } \\
\text { rule-set } \\
\text { - UX/UI (User Experi- } \\
\text { ence / User Interface) } \\
\text { for prompt and } \\
\text { integrated response } \\
\text { - Link information with } \\
\text { CCTVs and disaster } \\
\text { prevention and } \\
\text { management equip- } \\
\text { ment }\end{array}$ \\
\hline
\end{tabular}

\subsection{The scope of system interfaces}

The system interfaces are divided into three interface modules, as shown in Table 1.

The integrated information platform and the interface modules are designed to perform distributed processing and real-time information linking. In order to compensate for lost information, there are plans to develop the system, while taking into consideration equipment protection and data redundancy.

\section{CONCLUSION}

This paper provides a summary of the roles of the system interfaces in the integrated information platform developed for disaster response. While implementing this research project, the research group came across the following barriers:

1. In the case of fire notification information, which is communicated in the order of a sensor, receiver and alarm, there are limitations to linking it with the interface because the receiver information is kept closed to ensure stable fire alarm and management.

2. There is a need for a standard communication protocol [5] to integrate the information received from each sensor in the interface, but there are limitations in terms of access due to the issues of security and leakage of technology.

3. Even after the integrated information platform and system interface module are set up, there are additional stages such as the testing and certification process that remain. 
Once the integrated information platform described in this paper is established, big data analysis of the information obtained from diverse measuring systems can be performed for prompt and accurate decisions to be made during a disaster situation. At present, an integrated system of CPS [6] Level 2 is expected to result from this project, but a complete CPS that can seamlessly link the virtual world and the real world may be developed at a later time by incorporating artificial intelligence into the system.

\section{ACKNOWLEDGEMENTS}

This work was supported by the National Research Council of Science \& Technology (NST) grant by the Korea government (MSIP) (No. CRC-16-02-KICT)

\section{REFERENCES}

[1] Tae-Wook, K., Seung-Hwa, P. \& Chang-Hee, H., BIM/GIS-based data integration framework for facility management, GEOProcessing 2016 : The Eighth International Conference on Advanced Geographic Information Systems, Applications, and Services, pp. 100-105, 2016.

[2] Dianne, D., National BIM Standard: BIM GIS Integration, AEC Infosystems Presentation, 2007.

[3] Seung-Hwa, P., Chang-Hee, H. \& Youn-Mi, J., A study on an application of BIM/GIS interoperable platform for the implementation of SMART city. Proceeding of the KSCE 2016 Conference \& Civil Expo, pp. 127-128, 2016.

[4] Virtual Reality training software for safety and security; available at: http://www.xvrsim.com/?t=gb. (accessed 26 April, 2017).

[5] Integrated Proprietary Alarm Monitoring System, available at: http://www.digitize-inc. com. (accessed 26 April, 2017).

[6] Grant, C., Hamins, A., Bryner, N., Jones, A. \& Koepke, G., Research roadmap for smart fire fighting, pp. 6-13, 2015. 